\title{
UM ESTUDO TERMODINÂMICO DA CORROSÃO DOS AÇOS CARBONO PELO SULFETO DE HIDROGÊNIO - EXPLORANDO CONCEITOS DE EQUILÍBRIO QUÍMICO
}

\author{
Mariana Cristina de Oliveira, Alexandre Pereira de Lima, Rodrigo Monzon Figueredo, Heloisa Andréa Acciari* e Eduardo \\ Norberto Codaro \\ Departamento de Física e Química, Faculdade de Engenharia de Guaratinguetá, Universidade Estadual Paulista, 12516-410 \\ Guaratinguetá - SP, Brasil
}

Recebido em 04/09/2017; aceito em 07/12/2017; publicado na web em 18/01/2018

\begin{abstract}
A THERMODINAMIC CORROSION STUDY OF CARBON STEELS IN HYDROGEN SULFIDE - EXPLORING CHEMICAL EQUILIBRIUM CONCEPTS. This study addresses an inherent problem to the oil and natural gas exploration and production industry, the corrosion of pipes and tanks by $\mathrm{H}_{2} \mathrm{~S}$. $\mathrm{H}_{2} \mathrm{~S}$ with water emulsified in the oil or $\mathrm{H}_{2} \mathrm{~S}$ with the water vapor mixed in the gas, they make the acid environment leading to corrosion. This is commonly manifested by the formation of insoluble sulfides on the surface of the carbon steels destined for this purpose. The evolution of the corrosion process depends on the composition and physicochemical characteristics of sulfide film. In this context, we intend to interpret the conditions of formation and precipitation of iron sulfides as a function of $\mathrm{pH}$, using concepts of chemical equilibrium, electrode potential and electrochemical cell that are part of the subjects of chemistry.
\end{abstract}

Keywords: hydrogen embrittlement; sour corrosion; carbon steels; teaching corrosion.

\section{INTRODUÇÃO}

A corrosão dos aços-carbono de baixa liga pelo sulfeto de hidrogênio sempre foi um tema de pesquisa recorrente na área de exploração e produção de petróleo e gás natural, que adquiriu especial atenção depois da descoberta do pré-sal em 2006 no Brasil. Durante a recuperação primária, o fluido que sai espontaneamente dos poços de uma jazida a dezenas de atmosferas passa por um processo no qual o óleo cru, o gás e a água são separados. Em seguida, o óleo e o gás são bombeados para os terminais e daí para as refinarias e unidades de processamento, respectivamente. O sulfeto de hidrogênio está naturalmente presente no óleo e no gás e junto com a água emulsionada e o vapor de água tornam-se ambientes ácidos propícios para a corrosão. ${ }^{1-3}$ Deste modo, as tubulações para transferência e os tanques para armazenamento podem sofrer corrosão localizada ou mesmo generalizada, dependendo do aço, do fluido e das condições operacionais como pressão, temperatura e dinâmica do escoamento., ${ }^{4,5}$ Entre os tipos de corrosão localizada destaca-se a denominada fragilização pelo hidrogênio como a forma mais preocupante de corrosão. Neste processo, parte do hidrogênio que é reduzido durante a reação de corrosão penetra no aço e se acumula em falhas (descontinuidades + defeitos), as quais, após certa pressurização, levam à formação de trincas e, finalmente, à ruptura prematura do material. A corrosão generalizada manifesta-se pela formação de uma carepa de mono e polissulfetos de ferro nas paredes internas de dutos e válvulas ocasionando obstrução de fluxo e problemas nos sistemas de bombeamento. ${ }^{5}$ Embora as consequências da corrosão sejam conhecidas, as causas e os mecanismos pelos quais o fenômeno acontece ainda não estão bem elucidados. Dois fatores contribuem para esta situação: as composições químicas do petróleo, do gás natural e da água de formação variam de jazida para jazida e, a impossibilidade de reproduzir num laboratório as condições internas dos oleodutos, gasodutos e tanques.

Muitos profissionais ligados a companhias de petróleo dedicaram-se ao estudo dos processos corrosivos desses aços em presença de sulfetos e nesta missão a análise dos produtos de corrosão em dutos e

*e-mail: heloisa@feg.unesp.br reservatórios foi determinante no estudo retrospectivo do mecanismo da corrosão. A experiência em campo aliada à pesquisa científica permitiu discernir de certo modo quais daqueles produtos eram formados como consequência da corrosão e quais da contaminação do petróleo e do gás natural durante a produção. Comumente, uma tubulação ou um tanque apresenta uma camada mais ou menos contínua de óxidos e oxi-hidróxidos de ferro (hematita $\mathrm{Fe}_{2} \mathrm{O}_{3}$, magnetita $\mathrm{Fe}_{3} \mathrm{O}_{4}$, lepidocrocita $\gamma-\mathrm{FeOOH}$, goetita $\alpha-\mathrm{FeOOH}$ ) formada espontaneamente ao ar, ${ }^{6} \mathrm{a}$ qual não é removida antes do lançamento ou da instalação. Durante a passagem ou estocagem do fluido, o sulfeto de hidrogênio pode reagir com estes compostos, formar monossulfetos e separar enxofre elementar ou formar polissulfetos. Diferentes sulfetos de ferro foram identificados dentro de gasodutos e oleodutos. ${ }^{7-9}$ Os monossulfetos mais citados são a mackinawita tetragonal $(\mathrm{FeS})$, troilita hexagonal e as pirrotitas $\left(\mathrm{Fe}_{1-\mathrm{x}} \mathrm{S} \operatorname{com} \mathrm{x}=0\right.$ a 0,17$)$ e, os polissulfetos são a greigita $\left(\mathrm{Fe}_{3} \mathrm{~S}_{4}\right)$ e a pirita $\left(\mathrm{FeS}_{2}\right)$, principalmente esse último. ${ }^{10}$ Esses surgem como consequência da oxidação parcial dos monossulfetos por pequenas quantidades de oxigênio, possivelmente pelo ingresso de ar durante a recuperação secundária ou terciária do petróleo. Pesquisas empregando diferentes técnicas em meios menos complexos também tiveram uma contribuição significativa. Água do mar artificial e outras soluções salinas desaeradas, saturadas com sulfeto de hidrogênio, foram os meios corrosivos mais utilizados, provavelmente porque esses meios fazem referência à água de formação emulsionada no petróleo. ${ }^{11,12}$ Estudos revelaram que a mackinawita é formada rapidamente em uma ampla faixa de temperaturas e pressões parciais de $\mathrm{H}_{2} \mathrm{~S}$, é metaestável e precursora na formação de polissulfetos. A troilita é considerada o membro estequiométrico das pirrotitas e suas condições de formação não estão bem estabelecidas, geralmente, temperaturas e pressões parciais de $\mathrm{H}_{2} \mathrm{~S}$ menores que as necessárias para a formação das outras pirrotitas. As pirrotitas mais comuns, a hexagonal $\left(\mathrm{Fe}_{10} \mathrm{~S}_{11}\right)$ e a monoclínica $\left(\mathrm{Fe}_{7} \mathrm{~S}_{8}\right)$ nessa ordem, são muito estáveis em meios ácidos isentos de oxigênio. ${ }^{7,10,13}$ Resultados obtidos de ensaios de corrosão em tempos relativamente curtos de exposição à temperatura ambiente conduzem a um ou dois tipos de sulfetos de ferro, a mackinawita (forma cristalina cineticamente favorecida) e a pirrotita (forma cristalina termodinamicamente favorecida). Uma 
vez que a composição e as características físico-químicas do filme determinam o desenvolvimento da corrosão, no presente trabalho pretende-se interpretar as condições de formação e precipitação dessas espécies em função do $\mathrm{pH}$ utilizando conceitos de equilíbrio químico, potencial de eletrodo e célula eletroquímica.

\section{FUNDAMENTAÇÃO TEÓRICA}

\section{Sulfeto de hidrogênio em solução aquosa}

O sulfeto de hidrogênio é um gás relativamente solúvel em água à temperatura ambiente e a sua solubilidade é diretamente proporcional à sua pressão parcial de acordo com a Lei de Henry (equação 1).

$$
\mathrm{C}\left(\frac{\mathrm{mol}}{\mathrm{L}}\right)=0,1013 \frac{\mathrm{mol}}{\mathrm{L} \mathrm{atm}} \mathrm{pH}_{2} \mathrm{~S} \text { atm }
$$

Sendo C a solubilidade, $\mathrm{pH}_{2} \mathrm{~S}$ a pressão parcial e $0,1013 \mathrm{~mol} \mathrm{~L}^{-1}$ $\mathrm{atm}^{-1}$ a constante de Henry a $25^{\circ} \mathrm{C} .{ }^{14}$ Uma vez dissolvido, dissocia-se, comportando-se como um ácido fraco (equações 2 a 4) e, em consequência, para um determinado $\mathrm{pH}_{2} \mathrm{~S}$, a concentração real de $\mathrm{H}_{2} \mathrm{~S}_{(\mathrm{aq})}$ torna-se menor que $\mathrm{C}$, resultando mais apropriado estabelecer uma condição de equilíbrio a partir da pressão parcial.

$$
\begin{aligned}
& \mathrm{H}_{2} \mathrm{~S}_{(\mathrm{g})} \leftrightarrows \mathrm{H}_{2} \mathrm{~S}_{(\mathrm{aq})} \\
& \mathrm{H}_{2} \mathrm{~S}_{(\mathrm{aq})} \leftrightarrows \mathrm{H}^{+}{ }_{(\mathrm{qq})}+\mathrm{HS}^{-} \\
& \mathrm{HS}_{(\mathrm{aq})} \leftrightarrows \mathrm{H}^{+}{ }_{(\mathrm{aq})}+\mathrm{S}^{2-}{ }_{(\mathrm{aq})}
\end{aligned}
$$

Existe na literatura uma grande quantidade de valores para as constantes de dissociação deste ácido, em particular, para a segunda dissociação, a qual envolve a participação de concentrações de espécies iônicas muito baixas. ${ }^{15}$ Neste estudo foram adotados valores intermediários de $\mathrm{K}_{\mathrm{a} 1}=1,0 \times 10^{-7}$ e $\mathrm{K}_{\mathrm{a} 2}=1,0 \times 10^{-14}$ para o cálculo das concentrações no equilíbrio. Considerando os valores pequenos dessas constantes e a grande diferença entre elas, o $\mathrm{pH}$ de uma solução aquosa está determinado pela primeira dissociação (equação 5).

$$
\mathrm{pH}=-\log \sqrt{\mathrm{k}_{\mathrm{a} 1} \mathrm{C}}
$$

Como $\mathrm{C}$ depende de $\mathrm{pH}_{2} \mathrm{~S}$, combinando as equações 1 e 5 temos a relação que associa o pH com $\mathrm{pH}_{2} \mathrm{~S}$ (equação 6) e sua representação (Figura 1).

$$
\mathrm{pH}=4,0-0,5 \log \mathrm{pH}_{2} \mathrm{~S}
$$

Apesar das limitações da Lei de Henry, a Figura 1 permite expor duas características das soluções aquosas deste gás: i) em pressões ordinárias as soluções saturadas são levemente ácidas, ii) para mudar o pH em uma unidade é necessário alterar a pressão em duas ordens de magnitude.

Quando o pH da solução é modificado à pressão parcial e temperatura constantes, o equilíbrio é perturbado e uma nova relação de concentrações é estabelecida. Essa relação com o pH pode ser calculada a partir da expressão de $\mathrm{K}_{\mathrm{a} 1}$ para valores de $\mathrm{pH}$ menores do que 7,0 (equação 7) e da expressão da constante de hidrólise do ânion sulfeto $\left(\mathrm{K}_{\mathrm{h}}=1,0\right)$ para valores de $\mathrm{pH}$ maiores ou iguais a 7,0 (equação 8)

$$
\mathrm{pH}=7+\log \frac{\left[\mathrm{HS}_{(\mathrm{aq})}^{-}\right]}{\left[\mathrm{H}_{2} \mathrm{~S}_{(\mathrm{aq})}\right]}
$$

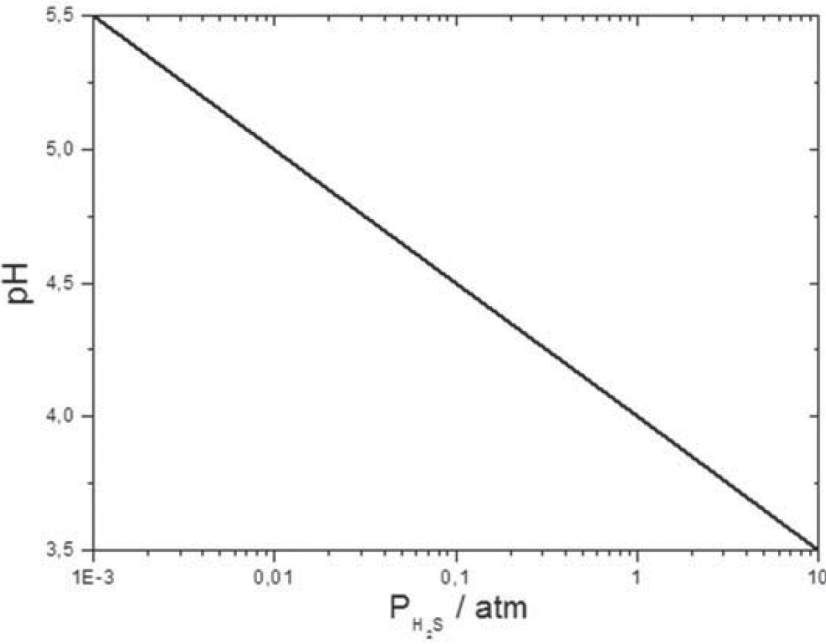

Figura 1. Variação do pH da solução com a pressão parcial de $\mathrm{H}_{2} \mathrm{~S}_{(\mathrm{g})}$ a $25{ }^{\circ} \mathrm{C}$

$$
\mathrm{pH}=14+\log \frac{\left[\mathrm{S}_{(\mathrm{aq})}^{2-}\right]}{\left[\mathrm{HS}_{(\mathrm{aq})}^{-}\right]}
$$

Na representação gráfica das equações 7 e 8 (Figura 2) pode-se observar a distribuição de $\mathrm{H}_{2} \mathrm{~S}_{(\text {aq)}}, \mathrm{HS}^{-}{ }_{(\text {aq) }} \mathrm{eS}^{2-}{ }_{(\text {aq) }}$, em função do $\mathrm{pH}$, para uma concentração total de espécies contendo enxofre de $0,1 \mathrm{~mol} \mathrm{~L}^{-1}$ determinada pela $\mathrm{pH}_{2} \mathrm{~S}=1,0$ atm a $25^{\circ} \mathrm{C}$.
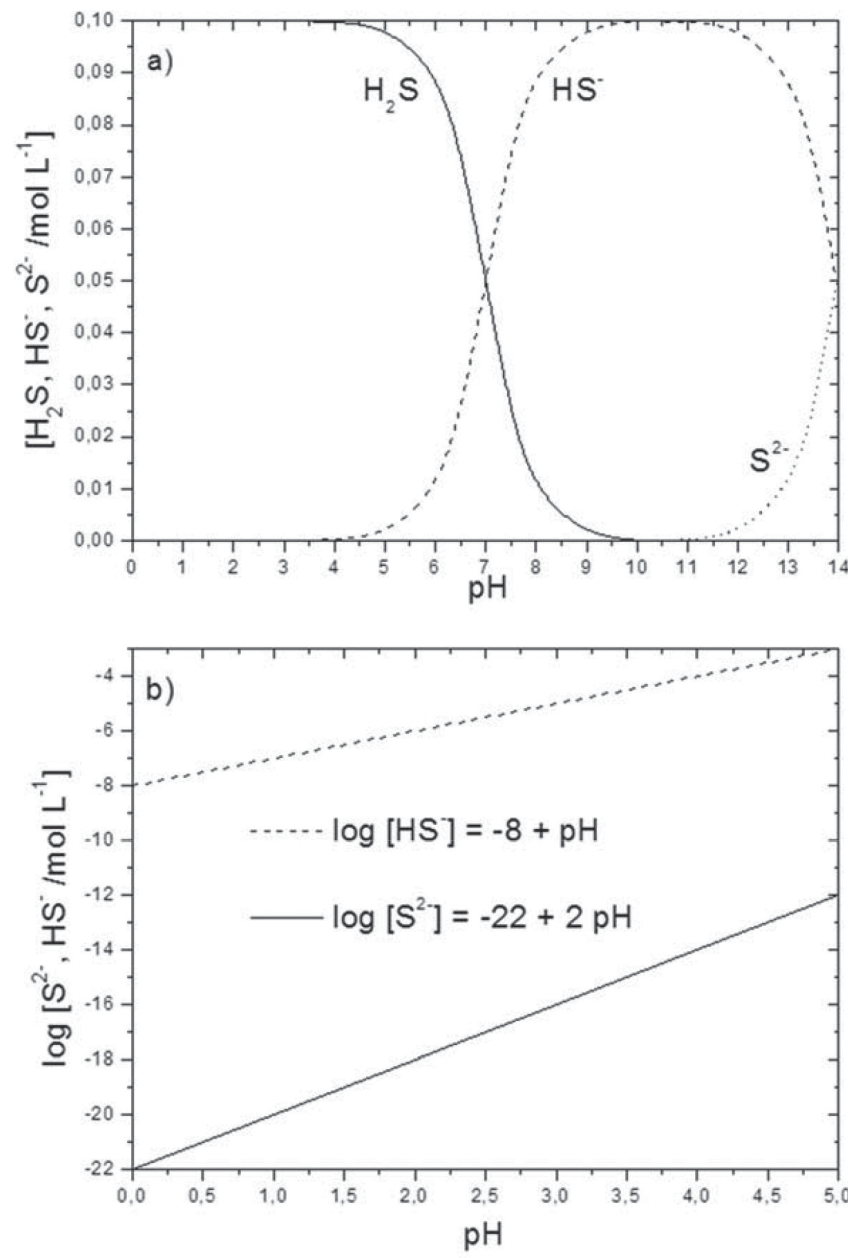

Figura 2. (a) Distribuição de espécies em solução aquosa de $H_{2} S_{(a q)}$ e (b) variação da concentração dos íons $H S_{(a q)}$ e $S_{(a q)}^{2-}$ em meio ácido 
A Figura 2a indica que, em soluções com valores de $\mathrm{pH}$ menores do que 4,0, o $\mathrm{H}_{2} \mathrm{~S}_{(\mathrm{aq})}$ está presente praticamente na forma molecular, mas mesmo nesta faixa existem pequenas concentrações de $\mathrm{HS}_{(\mathrm{aq})} \mathrm{e}$ $\mathrm{S}^{2-}{ }_{(\text {aq) }}$, prontas para reagir. Essas concentrações podem ser estimadas das expressões de $\mathrm{K}_{\mathrm{a} 1}$ e de $\mathrm{K}_{\mathrm{a} 1} \mathrm{~K}_{\mathrm{a} 2}$ (dissociação total), respectivamente, supondo $\left[\mathrm{H}_{2} \mathrm{~S}_{(\mathrm{aq})}\right]=0,1 \mathrm{~mol} \mathrm{~L}-1$. A Figura $2 \mathrm{~b}$ mostra em detalhe a variação da concentração dos íons $\mathrm{HS}_{(\text {aq) }}^{-}$e $\mathrm{S}^{2-}{ }_{(\mathrm{aq})}$ em meio ácido. Observa-se que em soluções fortemente ácidas, somente os sulfetos de metais de transição mais insolúveis podem precipitar. ${ }^{16}$

No diagrama de Pourbaix da Figura 3 são indicadas as espécies contendo enxofre, termodinamicamente estáveis, como uma função do potencial de eletrodo e do $\mathrm{pH}$ do meio. ${ }^{17}$ As linhas verticais correspondentes às soluções de $\mathrm{pH}=7,0$ e 14,0 representam as mesmas relações de concentração das espécies moleculares e iônicas no equilíbrio mostradas na Figura 2a. Duas linhas tracejadas foram adicionadas ao diagrama para indicar a região de estabilidade da água. As espécies que se situam dentro da região entre essas linhas não reagem com $\mathrm{H}_{2} \mathrm{O}$ e as que se situam fora podem reagir. Portanto, verifica-se que: i) $\mathrm{HSO}_{4}^{-{ }_{(a q)}}{\mathrm{e} \mathrm{SO}_{4}{ }_{4}{ }^{2-} \text { (aq) }}^{-}$ão estáveis em soluções aquosas em toda a faixa de $\mathrm{pH}$, ii) $\mathrm{S}_{(\mathrm{s})}$ é estável em soluções aquosas ácidas ou

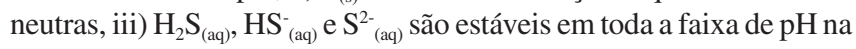
ausência de oxigênio, iv) a oxidação do $\mathrm{H}_{2} \mathrm{~S}_{(\mathrm{aq})}$ a $\mathrm{S}_{(\mathrm{s})}$ só pode acontecer em meio ácido na presença de oxigênio e como este é muito menos solúvel que o sulfeto de hidrogênio, ${ }^{14}$ torna-se o reagente limitante. Essa situação é mais acentuada quando a reação de corrosão de um aço ocorre em um sistema fechado, à medida que os íons $\mathrm{H}^{+}{ }_{(\mathrm{aq})}$ são consumidos, a pressão parcial do $\mathrm{H}_{2} \mathrm{~S}_{(\mathrm{g})}$ diminui enquanto que a do $\mathrm{H}_{2(\mathrm{~g})}$ aumenta, resultando em uma diferença cada vez maior entre os potencias de redução $\mathrm{E}\left(\mathrm{S} / \mathrm{H}_{2} \mathrm{~S}\right)$ e $\mathrm{E}\left(\mathrm{H}^{+} / \mathrm{H}_{2}\right)$.

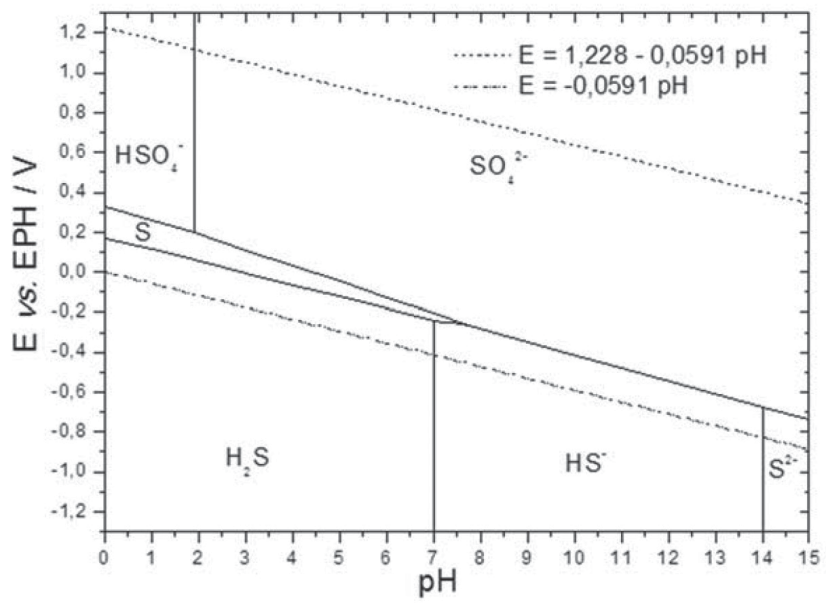

Figura 3. Diagrama de Pourbaix simplificado para o sistema $\mathrm{S}-\mathrm{H}_{2} \mathrm{O}$ a $25^{\circ} \mathrm{C}$, $\mathrm{pH}_{2} \mathrm{~S}=1,0 \mathrm{~atm}$, concentração total de espécies contendo enxofre $=0,1 \mathrm{~mol} \mathrm{~L}^{-1}$

\section{Ferro em solução aquosa}

A Figura 4 ilustra um diagrama de Pourbaix simplificado para o sistema $\mathrm{Fe}-\mathrm{H}_{2} \mathrm{O}$ a $25{ }^{\circ} \mathrm{C}$, o qual mostra entre linhas as regiões de estabilidade das espécies envolvidas. ${ }^{17}$ Este foi construído a partir de reações químicas e eletroquímicas possíveis associadas com o ferro em condições úmidas ou aquosas, excluindo aquelas que geram produtos secos como magnetita $\left(\mathrm{Fe}_{3} \mathrm{O}_{4(\mathrm{~s} s}\right)$ e hematita $\left(\mathrm{Fe}_{2} \mathrm{O}_{3(\mathrm{~s})}\right)$ e outros com insuficiente informação na literatura como os oxi-hidróxidos e oxiânions. Foi utilizada para os cálculos uma concentração de $\mathrm{Fe}^{2+}{ }_{\text {(aq) }} 100$ vezes maior do que a de $\mathrm{Fe}^{3+}{ }_{\text {(aq) }}$ por ser o primeiro o produto inicial da corrosão em meio ácido. A concentração de $\mathrm{Fe}^{3+}{ }_{\text {aq) }}$ foi fixada em $10^{-6} \mathrm{~mol} \mathrm{~L}^{-1}$, valor frequentemente encontrado nos ácidos minerais.

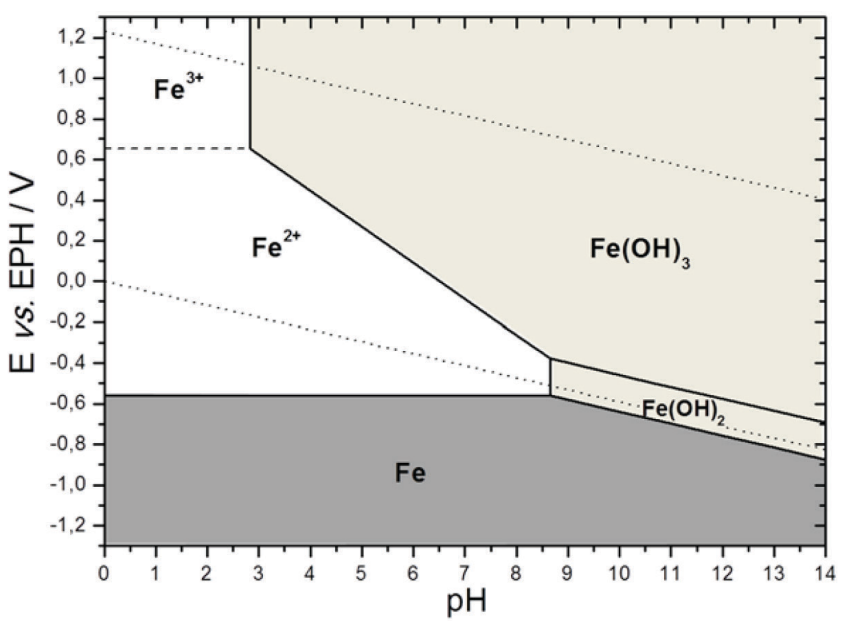

Figura 4. Diagrama de Pourbaix simplificado para o sistema $\mathrm{Fe}-\mathrm{H}_{2} \mathrm{O}$ a $25^{\circ} \mathrm{C}$, $\left[\mathrm{Fe}^{2+}\right]=10^{-4} \mathrm{~mol} \mathrm{~L}^{-1},\left[\mathrm{Fe}^{3+}\right]=10^{-6} \mathrm{~mol} \mathrm{~L} L^{-1}$

As duas linhas tracejadas sobre o diagrama encerram a região de estabilidade da água e uma reação de corrosão pode acontecer conforme o potencial de redução $\mathrm{Fe}^{2+}{ }_{(\mathrm{aq})} / \mathrm{Fe}_{(\mathrm{s})}$ é posicionado abaixo de uma dessas linhas. Na ausência de oxigênio, a reação catódica em meio ácido pode ser interpretada pela equação 9 e em meio neutro ou básico pela equação 10, enquanto que na presença de oxigênio, a reação em meio ácido pode ser representada pela equação 11 e em meio neutro ou básico pela equação 12 .

$$
\begin{gathered}
2 \mathrm{H}^{+}{ }_{\text {(aq) }}+2 \mathrm{e}^{-} \rightarrow \mathrm{H}_{2}(\mathrm{~g}) \\
2 \mathrm{H}_{2} \mathrm{O}_{(\mathrm{l})}+2 \mathrm{e}^{-} \rightarrow 2 \mathrm{OH}^{-}{ }_{\text {(aq) }}+\mathrm{H}_{2(\mathrm{~g})} \\
4 \mathrm{H}^{+}{ }_{(\mathrm{aq})}+\mathrm{O}_{2(\mathrm{aq})}+4 \mathrm{e}^{-} \rightarrow 2 \mathrm{H}_{2} \mathrm{O}_{(\mathrm{l})} \\
2 \mathrm{H}_{2} \mathrm{O}_{(\mathrm{l})}+\mathrm{O}_{2(\mathrm{aq})}+4 \mathrm{e}^{-} \rightarrow 4 \mathrm{OH}_{(\mathrm{aq})}^{-}
\end{gathered}
$$

Desse modo, o ferro pode ser oxidado em toda a faixa de $\mathrm{pH}$ e na ausência de oxigênio as espécies de ferro(II), $\mathrm{Fe}^{2+}{ }_{\text {(aq) }}$ (simplificação de $\left.\left[\mathrm{Fe}\left(\mathrm{H}_{2} \mathrm{O}\right)_{6}\right]^{2+}\right)$ e $\mathrm{Fe}(\mathrm{OH})_{2(\mathrm{~s})}$, são as mais estáveis. No entanto, cabe assinalar que com o incremento da pressão de hidrogênio a região de estabilidade da água aumenta e a elevadas pressões o ferro só pode ser oxidado em meio ácido. Quando o oxigênio está presente, o meio torna-se mais oxidante e essas espécies de $\mathrm{Fe}^{2+}$ são oxidadas $\mathrm{a} \mathrm{Fe}^{3+}{ }_{\text {(aq) }}\left(\right.$ simplificação de $\left.\left[\mathrm{Fe}\left(\mathrm{H}_{2} \mathrm{O}\right)_{6}\right]^{3+}\right), \mathrm{Fe}(\mathrm{OH})_{3(\mathrm{~s})}$ e aquocomplexos de ferro(III) não mostrados no diagrama. Apesar da duvidosa composição da ferrihidrita, $\mathrm{Fe}(\mathrm{OH})_{3},{ }^{18}$ esse composto será utilizado no lugar de $\mathrm{Fe}_{2} \mathrm{O}_{3} \cdot 3 \mathrm{H}_{2} \mathrm{O}_{\text {(s) }}$ para interpretar a precipitação seletiva de produtos de corrosão. Três regiões de estabilidade de espécies foram diferenciadas no diagrama: a sem cor, chamada domínio de corrosão (constituída pelos cátions), a cinza escura chamada domínio de imunidade (constituída pelo metal puro) e a cinza clara chamada domínio de passividade (constituída por um filme considerado, em princípio, protetor do metal). Essas regiões representam as condições teóricas onde pode, não pode e não acontece corrosão, respectivamente.

Por comparação das Figuras 3 e 4 deduz-se que o $\mathrm{H}_{2} \mathrm{~S}_{(\text {aq) }}$ pode ser oxidado por espécies de ferro(III), uma vez que o domínio do $\mathrm{H}_{2} \mathrm{~S}_{(\mathrm{aq})}$ não se superpõe aos domínios do $\mathrm{Fe}^{3+}{ }_{(\mathrm{aq})}$ e do $\mathrm{Fe}(\mathrm{OH})_{3(\mathrm{~s})}$.

\section{Ferro em ácido sulfídrico}

Quando a solução de sulfeto de hidrogênio entra em contato com o aço, uma reação de corrosão acontece e uma consequência pode ser a precipitação de sulfetos. Como a concentração das espécies iônicas deste meio está diretamente ligada ao $\mathrm{pH}$, a precipitação ou não de sulfetos como produto de corrosão do aço também dependerá do $\mathrm{pH}$. Existem na literatura diferentes expressões que determinam 
o equilíbrio de solubilidade e diversas constantes para esses equilíbrios. Neste estudo, as dependências da solubilidade com o $\mathrm{pH}$ foram deduzidas a partir das expressões convencionais dos produtos de solubilidade, ${ }^{16}$ considerando $\mathrm{K}_{\mathrm{ps} 1}=2,0 \times 10^{-15}$ para o $\mathrm{Fe}(\mathrm{OH})_{2}$ e $\mathrm{K}_{\mathrm{ps} 2}$ $=1,7 \times 10^{-19}$ para o FeS, equações 13 e 14, respectivamente. Como no caso do FeS o cálculo da equação de solubilidade envolve $K_{\mathrm{a} 2}$, alguns autores ${ }^{19-21}$ preferem usar outra expressão que não dependa dessa constante, comumente definida como limite de solubilidade (equação 15). No entanto, tanto uma como outra conduzem a resultados semelhantes para a finalidade deste estudo.

$$
\begin{gathered}
\mathrm{k}_{\mathrm{ps} 1}=\left[\mathrm{Fe}^{2+}\right]\left[\mathrm{OH}^{-}\right]^{2}=2,0 \times 10^{-15} \\
\mathrm{k}_{\mathrm{ps} 2}=\left[\mathrm{Fe}^{2+}\right]\left[\mathrm{S}^{2-}\right]=\left[\mathrm{Fe}^{2+}\right]\left(\frac{\mathrm{k}_{\mathrm{a} 1} \mathrm{k}_{\mathrm{a} 2}\left[\mathrm{H}_{2} \mathrm{~S}\right]}{\left[\mathrm{H}^{+}\right]^{2}}\right)=1,7 \times 10^{-19} \\
\mathrm{k}_{\mathrm{ps} 2 \mathrm{a}}=\frac{\left[\mathrm{Fe}^{2+}\right]\left[\mathrm{H}_{2} \mathrm{~S}\right]}{\left[\mathrm{H}^{+}\right]^{2}}=1,6 \times 10^{3}
\end{gathered}
$$

A Figura 5a representa a influência do $\mathrm{pH}$ na solubilidade de um $\mathrm{FeS}$ (mackinawita) em $\mathrm{H}_{2} \mathrm{~S}_{(\mathrm{aq})} 0,1 \mathrm{~mol} \mathrm{~L}^{-1}$ e do $\mathrm{Fe}(\mathrm{OH})_{2}$ em $\mathrm{H}_{2} \mathrm{O}$ a $25^{\circ} \mathrm{C}$, na ausência de $\mathrm{O}_{2}$. A figura revela que a concentração de $\mathrm{Fe}^{2+}{ }_{(\text {aq })}$ no equilíbrio diminui segundo o $\mathrm{pH}$ do meio aumenta em ambos os casos. Cada reta (equações 16 e 17) divide o gráfico em duas regiões: à esquerda, a região de dissolução e à direita, a região de precipitação.

$$
\begin{aligned}
& \log \left[\mathrm{Fe}^{2+}\right]=13,3-2 \mathrm{pH} \\
& \log \left[\mathrm{Fe}^{2+}\right]=3,22-2 \mathrm{pH}
\end{aligned}
$$

A precipitação de $\mathrm{FeS}$ inicia-se somente quando a concentração de $\mathrm{Fe}^{2+}{ }_{\text {(aq) }}$ e o $\mathrm{pH}$ encontram a reta e, quando isto acontece, o produto da concentração de $\mathrm{Fe}^{2+}{ }_{\text {(aq) }}$ pela concentração de $\mathrm{S}^{2-}{ }_{\text {(aq) }}$ ultrapassa o valor do $\mathrm{K}_{\mathrm{ps}}$. Torna-se evidente que a precipitação do $\mathrm{Fe}(\mathrm{OH})_{2}$ (ou $\mathrm{FeO}$. $\mathrm{H}_{2} \mathrm{O}$ ) no mesmo meio não acontece, uma vez que as concentrações $\mathrm{de} \mathrm{OH}_{(\mathrm{aq})}^{-} \mathrm{e} \mathrm{S}_{(\text {(aq) }}^{2-}$ guardam uma relação definida (Figura 2b) de modo que o produto iônico $\left[\mathrm{Fe}^{2+}\right]\left[\mathrm{OH}^{-}\right]^{2}$ é sempre menor que $\left[\mathrm{Fe}^{2+}\right]\left[\mathrm{S}^{2-}\right]$.

A Figura $5 \mathrm{~b}$ apresenta a influência do $\mathrm{pH}$ na solubilidade de um $\mathrm{FeS} \mathrm{em} \mathrm{H}_{2} \mathrm{~S}_{\text {(aq) }} 0,1 \mathrm{~mol} \mathrm{~L}^{-1}$ e do $\mathrm{Fe}(\mathrm{OH})_{3} \mathrm{em} \mathrm{H}_{2} \mathrm{O}$ a $25^{\circ} \mathrm{C}$. A expressão que representa a solubilidade do $\mathrm{Fe}(\mathrm{OH})_{3}$ foi obtida a partir de um valor intermediário do $\mathrm{K}_{\mathrm{ps} 3}=3,1 \times 10^{-40}$ (equação 18$) .{ }^{22}$

$$
\log \left[\mathrm{Fe}^{3+}\right]=2,49-3 \mathrm{pH}
$$

Destaca-se, ainda, que em meio ácido, o $\mathrm{Fe}(\mathrm{OH})_{3}$ é mais insolúvel que o FeS e, portanto, concentrações de $\mathrm{Fe}^{3+}{ }_{\text {(aq) }}$ muito menores que as de $\mathrm{Fe}^{2+}{ }_{\text {(aq) }}$ precipitam o $\mathrm{Fe}(\mathrm{OH})_{3}$. Isto sugere que se durante a reação de corrosão do aço uma pequena parte do $\mathrm{Fe}^{2+}{ }_{(\text {aq) }}$ é oxidado a $\mathrm{Fe}^{3+}{ }_{(\text {aq) }}$, existe a probabilidade de co-precipitação em certas condições de $\mathrm{pH}$. Por exemplo, a pH $=2,83$, se a concentração de $\mathrm{Fe}^{3+}{ }_{\text {(aq) }}$ for aproximadamente $0,01 \%$ da concentração molar de $\mathrm{Fe}^{2+}{ }_{\text {(aq) }}$ no equilíbrio, ambos $\mathrm{Fe}(\mathrm{OH})_{3}$ e $\mathrm{FeS}$ podem precipitar simultaneamente.

A dependência da solubilidade dos monossulfetos de ferro com $\mathrm{o} \mathrm{pH}$ à temperatura constante foi previamente estudada. ${ }^{13,21}$ Apesar das considerações realizadas no desenvolvimento deste estudo, a reta que descreve a solubilidade do FeS (Figura 5a) posiciona-se paralela entre as retas da troilita e da mackinawita tetragonal (Figura 5c). Nota-se também que a solubilidade de todos os monossulfetos diminui quando se aumenta o pH e que este fenômeno é menos acentuado nas pirrotitas, as mais insolúveis dos monossulfetos. Cabe destacar que as condições de nucleação e crescimento dessas fases são diferentes, ou seja, quando as condições locais favorecem a formação de uma determinada fase, todas as outras fases mais solúveis (maiores produtos de solubilidade) ou não se formam ou deixam de crescer caso alguma delas já foi formada em outras condições, por exemplo, durante a corrosão de aço, a mackinawita (forma cineticamente favorecida) deixa de crescer uma vez que as condições favorecem a formação de pirrotita, portanto, é de esperar a formação de mais uma camada.,
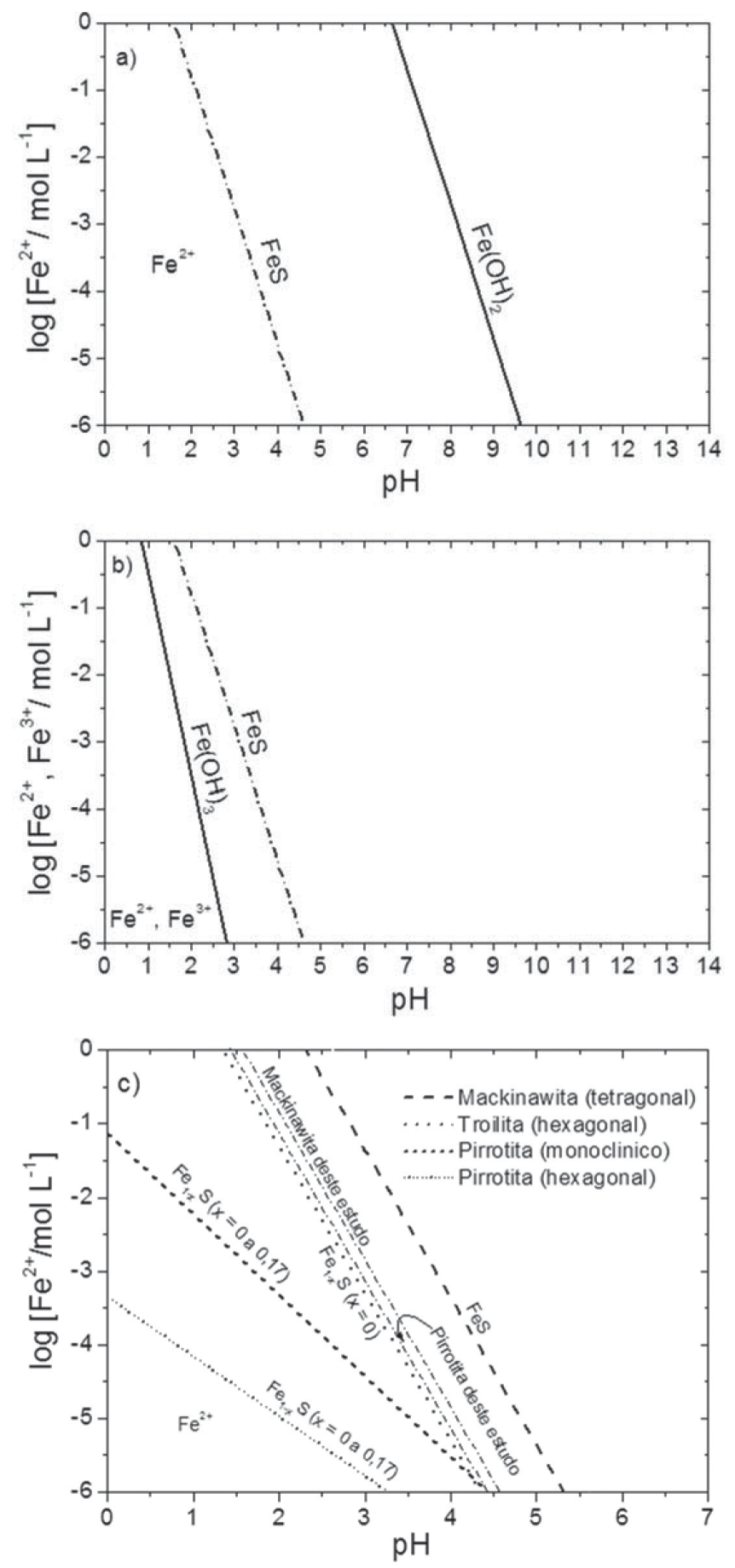

Figura 5. Influência do pH nas solubilidades: a) do $\mathrm{FeS}$ e $\mathrm{Fe}(\mathrm{OH})_{2}$, b) do $\mathrm{FeS}$ e $\mathrm{Fe}(\mathrm{OH})_{3}, \mathrm{c}$ ) dos monossulfetos de ferro em $\mathrm{H}_{2} \mathrm{~S}_{(a q)}, \mathrm{pH}_{2} \mathrm{~S}=1,0$ atm a $25^{\circ} \mathrm{C}$

Como não existe evidência experimental da transformação de fases entre a mackinawita e as pirrotitas, ao menos a temperaturas e pressões ordinárias, estas últimas serão formadas quando as condições do meio propiciarem a sua nucleação e crescimento, o que na prática 
ocorre em tempos prolongados de exposição e, provavelmente, em

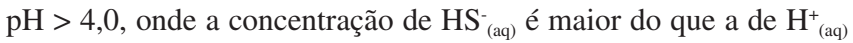
(Figura 2b). Para a construção do diagrama de Pourbaix com formação de pirrotitas (fases estáveis) também foi suposta uma concentração de $\mathrm{Fe}^{2+}{ }_{\text {(aq) }} 100$ vezes maior do que a de $\mathrm{Fe}^{3+}{ }_{\text {(aq) }}$ (Figura 6). Foram utilizadas as equações 18 e $19\left(\mathrm{~K}_{\mathrm{ps} 2 \mathrm{~b}}=79,4\right)^{21}$ para representar os equilíbrios químicos e as equações de Nernst 20 e 21, considerando $\Delta \mathrm{G}_{f}{ }^{0}\left(\mathrm{Fe}(\mathrm{OH})_{3}\right)=-708 \mathrm{~kJ} \mathrm{~mol}^{-1}$ e $\Delta \mathrm{G}_{f}^{0}(\mathrm{FeS})=-102 \mathrm{~kJ} \mathrm{~mol}^{-1}$ para os cálculos dos potenciais-padrão de redução $\mathrm{E}^{0}\left(\mathrm{Fe}(\mathrm{OH})_{3} / \mathrm{FeS}\right)^{18} \mathrm{e}$ $\mathrm{E}^{0}(\mathrm{FeS} / \mathrm{Fe}),{ }^{23}$ respectivamente. As linhas verticais representam os equilíbrios existentes entre as espécies de igual número de oxidação e correspondem às condições de equilíbrio determinadas pelas coordenadas $(2,83 ;-6)$ da Figura 5 b e $(3,45 ;-4)$ da Figura $5 c$.

$$
\begin{gathered}
\log \left[\mathrm{Fe}^{2+}\right]=2,90-2 \mathrm{pH} \\
\mathrm{E}(\mathrm{FeS} / \mathrm{Fe})=-0,354-0,0591 \mathrm{pH} \\
\mathrm{E}\left(\mathrm{Fe}(\mathrm{OH})_{3} / \mathrm{FeS}\right)=0,747-0,0591 \mathrm{pH}
\end{gathered}
$$

Assumindo a formação de um filme contínuo de sulfeto, observa-se que os domínios foram alterados se comparados com a Figura 4, ou seja, na Figura 6 o domínio de passivação é maior e o de corrosão menor o que pode ser interpretado como uma diminuição da área ativa devido à presença de um filme. Em recentes trabalhos, foram apresentados diagramas similares com formação da mackinawita e das pirrotitas, ${ }^{24-26}$ nos quais também foi demonstrado que o aumento da pressão parcial de $\mathrm{H}_{2} \mathrm{~S}(\mathrm{~g})$ favorece a formação dos monossulfetos de ferro. Este aumento desloca as retas da Figura $5 \mathrm{c}$ para esquerda (maiores $\mathrm{pH}$ ) e se traduz no diagrama como um aumento do domínio de passivação e a diminuição dos domínios de corrosão e imunidade. Uma análise mais detalhada desse diagrama revela que, em $\mathrm{pH}<3,45$, as condições não são favoráveis para a formação de filme e como o potencial de redução $\mathrm{Fe}^{2+}{ }_{(\mathrm{aq})} / \mathrm{Fe}_{(\mathrm{s})}$ é menor que o potencial de redução $\mathrm{H}^{+}{ }_{(\mathrm{aq})} / \mathrm{H}_{2(\mathrm{~g})}$, a reação de corrosão da equação 22 torna-se a mais provável na ausência de oxigênio. Quando o $\mathrm{pH} \geq 3,45$, o $\mathrm{Fe}^{2+}{ }_{\text {(aq) }}$ pode precipitar como $\mathrm{FeS}$ (equação 23) e formar um filme com certas características protetoras. As pesquisas indicaram que a precipitação de sulfetos acontece a $\mathrm{pH}$ $\geq 2,7$ e os filmes formados até $\mathrm{pH} \leq 4$ são descontínuos ou porosos, aqueles formados em $\mathrm{pH} \geq 6$ são compactos e, entre esses valores de $\mathrm{pH}$, possuem características intermediárias..$^{27-30}$

$$
\begin{aligned}
\mathrm{Fe}(\mathrm{s})+2 \mathrm{H}^{+}{ }_{(\mathrm{aq})} & \rightarrow \mathrm{Fe}^{2+}{ }_{(\mathrm{aq})}+\mathrm{H}_{2(\mathrm{~g})} \\
\mathrm{Fe}(\mathrm{s})+\mathrm{H}_{2} \mathrm{~S}_{(\mathrm{aq})} & \rightarrow \mathrm{FeS}_{(\mathrm{s})}+\mathrm{H}_{2(\mathrm{~g})}
\end{aligned}
$$

É importante esclarecer que o filme de sulfeto associado com a passivação não deve ser literalmente entendido como protetor fazendo com que a corrosão do aço não progrida. Na realidade, as propriedades físico-químicas do filme (aderência, compacidade, espessura e condutividade) em função do $\mathrm{pH}$ e do tempo de exposição determinarão as suas características protetoras.

\section{CONSIDERAÇÕES FINAIS}

Neste estudo foram explorados conceitos de equilíbrio químico, potencial de eletrodo e célula eletroquímica contextualizados na corrosão sulfídrica inerentes às indústrias de petróleo e gás natural. Foram levadas em conta as características do meio, o comportamento do material no meio e o processo corrosivo propriamente dito. As conclusões a seguir estão baseadas principalmente nos diagramas de Pourbaix e sujeitas às suas limitações, ou seja, não consideram aspectos cinéticos como mecanismo e velocidade de corrosão, propriedades dos filmes e nenhuma influência devido à presença de

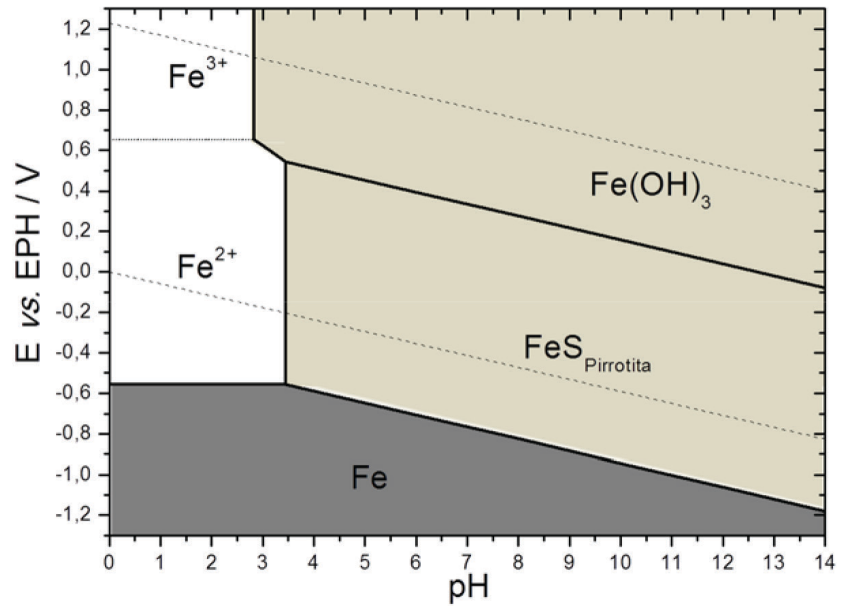

Figura 6. Diagramas de Pourbaix simplificados para o sistema $\mathrm{Fe}-\mathrm{H}_{2} \mathrm{O}-\mathrm{H}_{2} \mathrm{~S}$ a $25^{\circ} \mathrm{C}$ com formação de pirrotita. $\left[\mathrm{Fe}^{2+}\right]=10^{-4} \mathrm{~mol} \mathrm{~L}^{-1},\left[\mathrm{Fe}^{3+}\right]=10^{-6} \mathrm{~mol} \mathrm{~L}^{-1}$ e $\mathrm{pH}_{2} \mathrm{~S}=1,0 \mathrm{~atm}$

outros ânions e cátions. Além disso, o pH na interface dos eletrodos é teórico e não experimental à temperatura de $25^{\circ} \mathrm{C}$.

Neste contexto, durante a corrosão do aço carbono pelo ácido sulfídrico, é formado um filme de sulfeto ferroso (mackinawita), mais protetor que os óxidos e hidróxidos de ferro(II) formados sobre ferro em meio aquoso desaerado. As características protetoras do filme melhoram com a posterior formação de pirrotitas sobre a mackinawita. Apesar das limitações dos diagramas, a abordagem deste estudo em termos de equilíbrios de solubilidade dá suporte a um mecanismo de dissolução-precipitação para o crescimento de filmes. Óxidos, oxi-hidróxidos e hidróxidos de ferro(III), enxofre elementar, polissulfetos e sulfatos não estão entre os produtos de corrosão porque estes surgem como consequência da presença de oxigênio ou outros agentes oxidantes dissolvidos no meio.

\section{AGRADECIMENTOS}

PROEX/UNESP, CAPES e FAPESP (processo n. 2017/11361-5) pelos recursos financeiros.

\section{REFERÊNCIAS}

1. Tissot, B. P.; Welte, D. H.; Petroleum Formation and Occurrence, $2^{\text {nd }}$ ed., Springer-Verlag: Berlin, 1984, cap. 7.

2. Stumpf, A.; Tolvaj, K.; Juhász, M.; J. Chromatogr. A 1998, 67, 819.

3. Green, T. K.; Whitley, P.; Wu, K.; Lloyd, W. G.; Gan, L. Z.; Energy Fuels 1994, 8, 244

4. Liu, M.; Wang, J.; Ke, W.; Han, E. H.; J. Mater. Sci. Technol. 2014, 30, 504.

5. Mahmoud, M. A.; Kamal, M. S.; Geri, B. S. B.; Hussein, I. A.; Fahd, K.; Paper IPTC-18279-MS of International Petroleum Technology Conference, Doha, Qatar, 2015.

6. Silva, M. V. F.; Pereira, M. C.; Codaro, E. N.; Acciari, H. A.; Quim. Nova 2015, 38, 293.

7. Smith, S. N.; Brown, B.; Sun, W.; Paper 11081 of NACE International Conference \& Expo, Houston, USA, 2011

8. Craig, B.; Mater. Perform. 2002, August, 2.

9. Smith, S. N.; Mater. Perform. 2003, August, 44.

10. Morse, J. W.; Millero, F. J.; Cornwell, J. C.; Rickard, D.; Earth-Sci. Rev. 1987, 24, 1 .

11. NACE International; Standard test method laboratory testing of metals for resistance to sulfide stress cracking and stress corrosion cracking in $\mathrm{H}_{2} \mathrm{~S}$ environments. ANSI/NACE TM0177, Houston, USA, 2016. 
12. NACE International; Evaluation of Pipeline and Pressure Vessel Steel for Resistance to hydrogen-Induced Crack. ANSI/NACE TM0284, Houston, USA, 2016.

13. Rickard, D.; Luther, G.; Chem. Rev. 2007, 107, 514.

14. Sander, R.; Atmos. Chem. Phys. 2015, 15, 4399.

15. Sun, W.; Nesic, S.; Young, D.; Woollam, R. C.; Ind. Eng. Res. 2008, 47, 1738.

16. Skoog, D. A.; West, D. M.; Holler, J.; Crouch, S. R.; Fundamentos de Química Analítica, 9 ed., Cengage Learning: São Paulo, 2014, cap. 31.

17. Pourbaix, M.; Atlas of electrochemical equilibria in aqueous solutions, $2^{\text {nd }}$ ed.; NACE: Houston, 1974.

18. Majzlan, J.; Navrotsky, A.; Schwertmann, U.; Geochim. Cosmochim. Acta 2004, 68, 1049.

19. Myers, R. J.; J. Chem. Educ. 1986, 63, 687.

20. Rickard, D.; Geochim. Cosmochim. Acta 2006, 70, 5779.

21. Davison, W.; Aquat. Sci. 1991, 53/4, 309.

22. Schwertmann, U.; Plant Soil 1991, 130, 1.
23. Lennie, A. R.; Vaughan, D. J.; Special Publications - Geochemical Society 1996, 5, 117.

24. Ning, J.; Zheng, Y.; Young, D.; Brown, B.; Nesic, S.; Paper 2462 of NACE International Conference \& Expo, Orlando, USA, 2013.

25. Ning, J.; Zheng, Y.; Brown, B.; Young, D.; Nesic, S.; Paper 7502 of NACE International Conference \& Expo, Vancouver, Canada, 2016.

26. Ning, J.; Zheng, Y.; Brown, B.; Young, D.; Nesic, S.; Paper 5507 of NACE International Conference \& Expo, Dallas, USA, 2015.

27. Zheng, Y.; Ning, J.; Brown, B.; Young, D.; Nesic, S.; Paper 5933 of NACE International Conference \& Expo, Dallas, USA, 2015.

28. Shoesmith, D. W.; Taylor, P.; Bailey, M. G.; Owen, D. G.; J. Electrochem. Soc.: Electrochem. Sci. Technol. 1980, May, 1007.

29. Smith, S. N.; Paper 5485 of NACE International Conference \& Expo, Dallas, USA, 2015.

30. Wikjord, A. G.; Rummery, T. E.; Doern, F. E.; Owen, D. G.; Corros. Sci. 1980, 20, 651 . 